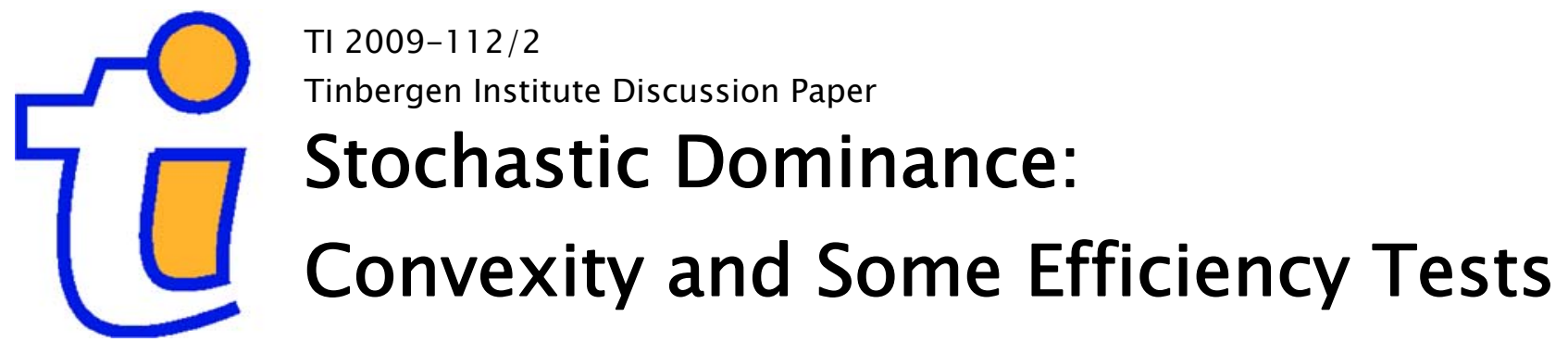

Andrey M. Lizyayev

Erasmus School of Economics, Erasmus University, and Tinbergen Institute. 


\section{Tinbergen Institute}

The Tinbergen Institute is the institute for economic research of the Erasmus Universiteit Rotterdam, Universiteit van Amsterdam, and Vrije Universiteit Amsterdam.

Tinbergen Institute Amsterdam

Roetersstraat 31

1018 WB Amsterdam

The Netherlands

Tel.: +31(0)205513500

Fax: $+31(0) 205513555$

Tinbergen Institute Rotterdam

Burg. Oudlaan 50

3062 PA Rotterdam

The Netherlands

Tel.: + $31(0) 104088900$

Fax: $+31(0) 104089031$

Most TI discussion papers can be downloaded at http://www.tinbergen.nl. 


\title{
Stochastic Dominance: Convexity and Some Efficiency Tests*
}

\author{
Andrey Lizyayev ${ }^{\dagger}$ \\ December 9, 2009
}

\begin{abstract}
This paper points out the importance of Stochastic Dominance (SD) efficient sets being convex. We review classic convexity and efficient set characterization results on SD efficiency of a given portfolio relative to a diversified set of assets and generalize them in the following aspects. First, we broaden the class of individual utilities in Rubinstein (1974) that lead to two-fund separation. Secondly, we propose a linear programming SSD test that is more efficient than that of Post (2003) and expand the SSD efficiency criteria developed by Dybvig and Ross (1982) onto the Third Order Stochastic Dominance and further to Decreasing Absolute and Increasing Relative Risk Aversion Stochastic Dominance. The efficient sets for those are finite unions of convex sets.
\end{abstract}

JEL classification: G11, C63.

KEYWORDS: Stochastic Dominance, Convexity, Risk Aversion, Efficiency.

*I would like to thank Prof. Dr. T. Kuosmanen from Helsinki School of Economics and Prof. Dr. T. Post, Prof. Dr. W. Verschoor and Dr. P. Versijp from Erasmus University, Rotterdam, for their helpful comments and valuable discussions.

${ }^{\dagger}$ Erasmus School of Economics, Erasmus University Rotterdam, PO Box 1738, 3000 DR Rotterdam, lizyayev@ese.eur.nl 


\section{Introduction}

Stochastic Dominance (SD) is a probabilistic concept of superiority among different random variables. Unlike parametric criteria such as Mean-Variance analysis, SD accounts for the whole range of distribution function, rather than its particular characteristics such as central moments. Although SD has applications in a huge variety of areas ranging from medicine to agriculture (see, e.g., Bawa 1982 and Levy 1992, 2006 for a survey and references, Eeckhoudt et al 2009 for recent applications), this paper focuses on its use in the area of finance. In financial decision-making one has to select efficient portfolios from an available portfolio possibilities set on the basis of a trade-off among their expected returns, the associated risk of having extreme losses and the potential of earning excessive gains.

We consider the expected utility framework whereby individuals select portfolios maximizing the expected value of their utility function which can capture different individual risk attitudes such as risk aversion, risk neutrality, risk seeking, or a combination of those, for different levels of wealth. The non-parametric nature of SD criteria allows us to identify efficient portfolios without having to specify the utility functions explicitly. Instead, it employs some general restrictions such as non-satiation and risk aversion. The set of all portfolios supported by some utility function in a given class is called the efficient set with respect to this utility class. It turns out that convex efficient sets have a special economic content and hence necessary and sufficient conditions leading to the convexity of efficient sets have been puzzling researchers for more than three decades. Rubinstein (1974) showed that when the preferences of all investors are similar enough, two-fund separation results. On the other hand, Dybving and Ross (1982) proved that if no assumptions on investors' preferences are made other than concavity and monotonicity, the efficient set is generally non-convex. In line with this concave and monotone utility class Ross (1978) derived some assumptions on the distribution function of returns that lead to k-fund separation. Among recent researchers, Versijp (2007) reviewed Rubinstein's result in relation to stochastic dominance and asset pricing models.

In this paper we point out the importance of Stochastic Dominance efficient sets being convex, review classic convexity and efficient set characterization results and generalize them in the following aspects. First, we broaden the class of individual utilities in Rubinstein (1974) that lead to two-fund separation. Second, we expand the SSD efficiency criteria developed by Dybvig 
and Ross (1982) onto the Third Order Stochastic Dominance and further to Decreasing Absolute and Increasing Relative Risk Aversion Stochastic Dominance. The efficient sets for those are finite unions of intersections of convex sets. We also give a linear programming SSD efficiency test which is more efficient than that of Post (2003) in case of unrestricted short sales.

This paper is focused on portfolio efficiency with respect to a diversified portfolio possibilities set, normally a polytope whose vertices are the assets available to investors. Further, we consider discrete distribution of returns due to its interpretability via empirically observed data, as well as tractability of the computational methods involved.

This paper is organized as follows: Section 2 provides general assumptions and problem formulation, Section 3 points out the importance of efficient sets being convex and reviews the associated necessary and sufficient conditions, Section 4 suggests some efficiency tests for a given portfolio relative to various economically meaningful classes of utility functions, and finally Section 5 summarizes the major results and concludes the paper.

\section{Assumptions and problem formulation}

Consider a single period investment decision-making problem under uncertainty in a classic expected utility framework, in which:

1. Investors select investment portfolios to maximize the expected utility of the return on their investment portfolio. Let $U=\{u: \mathbb{R} \rightarrow \mathbb{R}\}$ denote the class of von Neuman-Morgenstern utility functions and $X$ be the $m$-by- $n$ matrix of returns of $n$ available assets in $m$ states of the world. The probability of occurrence of state $i$ is denoted $\pi_{i}$. Naturally, $0<\pi_{i} \leq 1, i=1, \ldots, m$, and $\sum_{i=1}^{m} \pi_{i}=1$. Investors are uncertain about which of the states of nature will occur, but they know the underlying probabilities of the states with certainty.

2. Investors may diversify between available assets. Denote $\lambda \in \Lambda$ for a vector of portfolio weights. Unless otherwise specified, we assume that short sales are allowed and unrestricted. The portfolio possibilities set then becomes

$$
\Lambda=\left\{\lambda \in \mathbb{R}^{n}: \lambda^{\top} e=1\right\}
$$

and the set of all available allocations is

$$
M_{X}=\left\{x \in \mathbb{R}^{m}: x=X \lambda, \quad \lambda \in \Lambda\right\}
$$


3. If a riskless asset is available in the market, it will be used either as part of $X$ (a column with equal components), or separately (in which case $X$ will be the set of risky assets only), whichever is more convenient.

A given portfolio $\tau \in \Lambda$ is optimal for an investor with utility $u \in U$ if and only if

$$
E u(X)=\sup _{\lambda \in \Lambda} E u(X \lambda)
$$

where $E u$ denotes the expected value of $u$.

If $\pi_{1}, \ldots, \pi_{m}$ are probabilities of occurrence of the states of the world, then (1) becomes

$$
\sum_{i=1}^{m} \pi_{i} u\left(x^{i} \lambda\right)
$$

where $x^{i}$ is the $i$-th row of $X$. In practical applications full information about utility functions is not available, and (2) cannot be verified directly. This provides the rationale for relying on a set of general assumptions, rather than a full specification of the utility function. The Second Order Stochastic Dominance criterion (SSD) restricts attention to the class of strictly increasing and concave utility functions, modeling thereby non-satiable and risk-averse preferences. The Third Order SD (TSD) assumes that in addition to SSD utilities are positively skewed. A portfolio $\tau \in \Lambda$ is said to be optimal in a given utility class $U$ if and only if there exists $u \in U$ such that $\tau$ is optimal for $u$ in the sense of (1).

A portfolio $\tau \in \Lambda$ is efficient if it is not dominated by any other portfolio, that is

$$
\forall \lambda \in \Lambda \backslash\{\tau\} \quad \exists u \in U: E u(X \lambda)<E u(X \tau) .
$$

If both $\Lambda$ and $U$ are convex (as will be the case in this paper), efficiency is equivalent to optimality due to the Minimax theorem (see e.g. Post (2003)) and thus the two concepts will henceforth be used interchangeably.

An individual investor with utility $u \in U$ is facing the following portfolio allocation problem:

$$
\max _{\lambda} \sum_{i=1}^{m} \pi_{i} u\left(x^{i} \lambda\right)-\nu\left(\lambda^{\top} e-1\right)
$$

where $\nu$ is the Lagrange multiplier. It is known that linearity constraints do not alter convexity. So if we assume $u(x)$ to be strictly concave and 
twice continuously differentiable in $x$, it will remain concave in $\lambda$. In fact, the Hessian of $u$ with respect to $\lambda$ is $H u=-Y^{\top} Y$, where $Y$ is an $m$-by-n matrix defined by $y_{i j}=-x_{i j} \sqrt{-u^{\prime \prime}\left(x^{i} \lambda\right)}$. Therefore, $H u$ is always negative semidefinite, and $u$ is concave in $\lambda$.

In light of the above-mentioned, the necessary and sufficient condition for $\tau$ to be the solution of (3) is that there exists $v \in \mathbb{R}$ such that for all $j=1 \ldots n$

$$
\sum_{i=1}^{m} \pi_{i} x_{i j} u^{\prime}\left(x^{i} \tau\right)=\nu
$$

Note that if a risk-free asset is available, due to (4) there should hold: $\sum_{i=1}^{m} \pi_{i} u^{\prime}\left(x^{i} \tau\right)=\frac{\nu}{r_{F}}$, where $r_{F}$ is the risk-free return. Thus, the optimality condition (4) takes on the form:

$$
\text { for all risky assets } j: \sum_{i=1}^{m} \pi_{i}\left(x_{i j}-r_{F}\right) u^{\prime}\left(x^{i} \tau\right)=0 \text {. }
$$

We could also relax the twice continuous differentiability assumption for $u$; (4) would still hold in optimality if $u$ is substituted by $\partial u$ - any vector from the supergradient correspondence.

\section{Convexity}

Program (3) represents the portfolio formation of an individual investor having a particular well-behaved utility function $u$. In macroeconomic settings, the aggregate investment decision of a large group of individuals, assuming all of them to be well-behaved, for instance non-satiable risk averters, is of primary importance. A reasonable theoretical model should allow us to extract information about all investors' decisions based on a small number of large composite portfolios. The largest of those, the total value-weighted aggregate portfolio, is generally referred to as the market portfolio, the efficiency of which has been a starting point of many asset pricing theories, including the capital asset pricing model (CAPM).

The simplest case when the market portfolio is efficient is two-fund separation, where any optimal portfolio is a linear combination of two assets, normally a risk-free asset and the market portfolio. In such an economy any individual investor will hold a share of the same (risky) market portfolio and will invest the rest of his/her wealth in the risk-free asset available, i.e. 
either borrowing or lending at the risk-free rate. Various assumptions lead to two-fund separation, such as: mean-variance setting (when investment decision is a trade-off only between mean returns and variances of underlying portfolios, see Markowitz $(1952,1987)$ ), homogeneity of preferences (see Rubinstein (1974)), joint normal distribution of asset returns, which is a common assumption of the traditional Capital Asset Pricing Model (CAPM, see Cochrane (2005) for an overview) and quadratic utility functions.

Despite its theoretical appeal, two-fund separation is extremely restrictive and is very unlikely to hold in practice. A straightforward generalization of the concept, preserving the market portfolio efficiency, is $k$-fund separation, where each efficient portfolio is a linear combination of $k$ fixed mutual funds. Naturally, $k$-fund separation is of practical and theoretical interest only when $k \ll n$. Generally $k$-fund separation holds in complete markets (see e.g. Dybving and Ross (1982)). Ross (1978) derives a necessary and sufficient condition for $k$-fund separation which however involves returns only; the result is hard to generalize on possible variation of individual preferences and is therefore not particularly informative as far as variations in utility functions are concerned. ${ }^{1}$

A natural further generalization of $k$-fund separability is the convexity of efficient sets. Indeed, the market portfolio is nothing other than a convex combination of all individual portfolios (with unknown positive weights), and therefore the convexity of an efficient set suffices for the efficiency of the corresponding market portfolio. Indeed, we may assume without loss of generality that individual assets are optimal for at least one investor with a well-behaved utility function and that therefore those assets are efficient. (If, however, we do have an asset whose returns are strictly dominated by another marketed asset or fund, we may as well discard it, as no rational investor will invest in it). Clearly, the market portfolio is now an interior point of a polyhedron whose vertices are all efficient, and if the whole efficient set is known to be convex, efficiency of the market portfolio automatically follows. In addition to being an implication of various asset pricing theories, efficiency of the market portfolio has an intuitive economic interpretation. Observing the popularity of large composite index funds (which proxy the market portfolio) among many individual and institutional investors in practice, many

\footnotetext{
${ }^{1}$ In fact, Theorem 3 in Ross (1978) can be seen as a refinement of the classic definition of $k$-fund separability, as both are given solely in terms of returns and both assume $k$ generating factors.
} 
researchers argue that even heterogeneous investors models inconsistent with two-fund separation should imply efficiency of the market portfolio. Moreover, 2- and $k$-fund separation are merely particular cases of efficient sets being convex.

Conditions which lead to convexity of efficient sets have been challenging researchers for more than three decades already, as it could deliver interesting aggregation results for the models of heterogeneous investors. If an economy is close to satisfying $k$-fund separation, there is no need for active investment, as every investor is better off investing into $k$ available mutual funds (with specific allocation among those funds determined individually for each investor) and saving on transaction costs associated with actively trading strategies. The cases when $k$-fund separation does not hold but the efficient set is convex are still of theoretical interest, as one could study utility preferences that support large composite portfolios, or test implications of heterogeneous investors models, refining the utility class on the basis of observed individual allocations and composite market indices.

The convexity puzzle can be tackled from two different perspectives: returns on underlying assets and preferences of individual investors. The former would lead the reader towards arbitrage pricing theories and various factor models, while the latter remains not duly researched. Dybvig and Ross (1982) do show with a simple example that SSD efficient set is generally non-convex. However, an SSD efficient set comprises portfolios optimal for all non-satiable risk-averse preferences, many of which are known to be unrealistic. For that reason, after reviewing and providing a more constructive proof of the results in Rubinstein (1974) related to 2-fund separation, we shall summarize the result of Dybvig and Ross (1982) and give efficiency tests for some refined utility classes containing far less unrealistic preferences than all risk averters.

\subsection{Homogeneous preferences}

Rubinstein (1974) considers three heterogeneous investors models in which individual preferences are modeled according to the following utility func- 
tions:

$$
\begin{aligned}
& u(x) \sim-\exp \left(-\frac{x}{A}\right), \quad A>0 \\
& u(x) \sim-\ln (A+x), \quad A>0 \\
& u(x) \sim \frac{(A+B x)^{(1-b)}}{1-b}, \quad A>0, B>0, b>0, b \neq 1
\end{aligned}
$$

Rubinstein shows that two-fund separation results if all agents have the same taste parameters $B$ and beliefs $\pi$, but may have different parameters $A$ in (6a) and (6b), and $A$ and $B$ in (6c). He assumes availability of a risk-free asset and requires in addition that $B=\frac{1}{b}$ in (6c). Below we sketch a more constructive proof of two-fund separation than the original one of Rubinstein and show that varying $B$ 's across individuals will not alter the two-fund separation, provided that the agents have the same power parameter $b$, even if $B \neq \frac{1}{b}$, thereby generalizing the result of Rubinstein.

Since all the functions above are strictly concave and twice continuously differentiable, a sufficient and necessary condition for portfolio optimality is (4). Let $r_{F}$ be the risk free rate, and $X$ - all risky asset returns. It is convenient to split the portfolio into its risk-free investment $\alpha$ and the remaining risky part $(1-\alpha)$. The portfolio allocation program now becomes

$$
\max _{\lambda \in \Lambda} \sum_{i=1}^{m} \pi_{i} u\left(1+\alpha r_{F}+(1-\alpha) x^{i} \lambda\right)-\nu\left(\lambda^{\top} e-1\right)
$$

The optimality conditions (4) are now:

$$
\left\{\begin{array}{l}
\sum_{i=1}^{m} \pi_{i} x_{i j}(1-\alpha) u^{\prime}\left(1+\alpha r_{F}+(1-\alpha) x^{i} \tau\right)=\nu, \quad \forall j \in \mathcal{N} \\
\sum_{i=1}^{m} \pi_{i}\left(r_{F}-x^{i} \tau\right) u^{\prime}\left(1+\alpha r_{F}+(1-\alpha) x^{i} \tau\right)=0
\end{array}\right.
$$

Let us start with the exponential utility class (6a). Suppose a portfolio $\left(\alpha_{1},\left(1-\alpha_{1}\right) \tau\right)$ is optimal for $u\left(x, A_{1}\right)$. By (8), this happens if and only if

$$
\left\{\begin{array}{l}
\sum_{i=1}^{m} \pi_{i} x_{i j}\left(1-\alpha_{1}\right) \exp \left(-\frac{1+\alpha_{1} r_{F}+\left(1-\alpha_{1}\right) x^{i} \tau}{A_{1}}\right)=\nu_{1}, \quad \forall j \in \mathcal{N} \\
\sum_{i=1}^{m} \pi_{i}\left(r_{F}-x^{i} \tau\right) \exp \left(-\frac{1+\alpha_{1} r_{F}+\left(1-\alpha_{1}\right) x^{i} \tau}{A_{1}}\right)=0
\end{array}\right.
$$


One can check by straightforward substitution to (9) that for any $A_{2}>0$ the optimal portfolio for the investor with utility $u\left(x, A_{2}\right)$ will be $\left(\alpha_{2},\left(1-\alpha_{2}\right)\right)$, with $\alpha_{2}=1-\frac{A_{2}}{A_{1}\left(1-\alpha_{1}\right)}$. This proves that the efficient portfolio corresponding to $u\left(x, A_{2}\right)$ has the same composition of risky assets. Due to the uniqueness of the solution to (9), and continuity of $f\left(A_{2}\right)=1-\frac{A_{2}}{A_{1}\left(1-\alpha_{1}\right)}$ as a function of $A_{2}$, two-fund separation follows.

Note that we can make derivations above only if $\alpha_{1} \neq 1$, that is, not all the budget is invested in the riskless asset. The portfolio $(\alpha=1,0)$ will not be optimal for any agent $u(x, A)$ with $A>0$, except in cases where $X$ happens to satisfy the second equation in (9) for $\alpha=1$. However, the risk-free asset will always be asymptotically efficient as risk aversion increases.

One can similarly check by substitution that $\left(\alpha_{2}, 1-\alpha_{2}\right)$, where

$$
\alpha_{2}=\frac{A_{1}+\alpha_{1}\left(1+r_{F}\right)-A_{2}\left(1-A_{1}\right)}{A_{1}+1+r_{F}},
$$

is optimal for $u(x)=-\ln \left(A_{2}+x\right)$, whenever $\left(\alpha_{1}, 1-\alpha_{1}\right)$ is optimal for $u(x)=-\ln \left(A_{1}+x\right)$.

Analogously, as soon as $\left(\alpha_{1}, 1-\alpha_{1}\right)$ satisfies (8) for $u(x)=\frac{1}{1-b}\left(A_{1}+B_{1} x\right)^{(1-b)}$ with Lagrangean $\nu=\nu_{1}$, optimality conditions $(8)$ for $u(x)=\frac{1}{1-b}\left(A_{2}+B_{2} x\right)^{(1-b)}$ will hold with the optimal portfolio $\left(\alpha_{2}, 1-\alpha_{2}\right)$, where

$$
\alpha_{2}=\frac{A_{2} B_{1}\left(\alpha_{1}-1\right)+B_{2} B_{1}\left(1+r_{F}\right)+A_{1} B_{2}}{B_{2}\left(r_{F} B_{1}+A_{1}+B_{1}\right)},
$$

and new Lagrangean multiplier

$$
\nu_{2}=\nu_{1}\left(\frac{1-\alpha_{2}}{1-\alpha_{1}}\right)^{1-b}\left(\frac{B_{2}}{B_{1}}\right)^{-b} .
$$

This proves the two-fund separation for homogeneous utility functions of the form (6a), (6b) and (6c).

Note, that this proof generalizes Rubinstein's result, as utility functions in (6c) are allowed to have different taste parameters ( $B$ 's) now, provided they agree on the power parameter $b$. The restriction $B=\frac{1}{b}$ explicitly imposed in Rubinstein (1974) can be omitted without distorting the two-fund separation. Note also that one explicit assumption behind the derivations above is that the number of underlying assets (including the riskless one) is less than or equal to the number of states: $n+1 \leq m$. 


\section{Generalizing preferences: non-convexity and some higher order efficiency tests}

So far we have analyzed the set of utilities leading to two-fund separation. Although this is a particular case of convex SD efficient sets, it only allows for homogeneous utilities among all investors in the sense that the preferences of all investors are assumed to be parameterized by one or two single parameters, which implies that investors have very similar tastes and as a result take similar investment decisions. Therefore we would like to broaden the class of individual utility functions to allow for heterogeneity among investors. The question is whether the efficient sets for those extended utility classes remain convex. Consider first the set of all risk-averse and non-satiable investors.

Dybvig and Ross (1982) give a simple example of a non-convex second order Stochastic Dominance (i.e. when $U=U_{2} \equiv\{u: \mathbb{R} \rightarrow \mathbb{R}$ : $\left.\left.u^{\prime}(x)>0, u^{\prime \prime}(x)<0, \forall x \in \mathbb{R}\right\}\right)$ efficient set with $n=3$ assets and $m=4$ states. They state the following necessary and sufficient conditions for SSD efficiency of portfolio $x^{0}$.

An allocation $x^{0} \in M_{X}$ is efficient in $U_{2}$ if and only if there exists $z^{0} \in \mathbb{R}^{m}$ such that:

$$
\begin{aligned}
& \text { (i) } x^{\top} z^{0} \text { is constant for all } x \in M_{X} \\
& \text { (ii) } x_{i}^{0}<x_{j}^{0} \Rightarrow \frac{z_{i}^{0}}{\pi_{i}} \geq \frac{z_{j}^{0}}{\pi_{j}}, \quad \forall i, j \\
& \text { (iii) } z^{0}>0
\end{aligned}
$$

Vector $z^{0}$ can be interpreted as a vector of marginal utility rationalizing portfolio $x$.

Condition (i) holds only if short sales are allowed and unrestricted. Otherwise (i) holds only for strictly interior points. In general (i) reads: $\left(x^{0}\right)^{\top} z^{0} \geq x^{\top} z^{0}, \forall x \in M_{X}$. Conditions (ii) and (iii) reflect the existence of a strictly concave supporting utility function and the inequality sign may be changed, depending on the properties of the utility class considered. For instance, for strictly concave functions there should hold: $\frac{z_{i}^{0}}{\pi_{i}} \geq \frac{z_{j}^{0}}{\pi_{j}} \Rightarrow x_{i}^{0} \leq x_{j}^{0}$; if the functions are in addition differentiable then $\frac{z_{i}^{0}}{\pi_{i}}>\frac{z_{j}^{0}}{\pi_{j}} \Rightarrow x_{i}^{0}<x_{j}^{0}$.

The non-convexity example of Dybvig and Ross is both disappointing and challenging. It shows on the one hand that even relative to the set of rather well-behaved preferences the market portfolio can be inefficient. On the other 
hand, the result challenges us to examine more refined utility classes - after all, if the utility set is restricted to nearly-homogeneous investors as in Rubinstein (1974), not only convexity follows, but even two-fund separation. Taking this into account, below we derive efficiency tests for some higher order SD criteria. In that same section we analyze the case when short sales are allowed and unrestricted, for the following two reasons. Although by far the majority of efficiency tests published assume away short sales, the efficiency of a given portfolio in the unrestricted case implies its efficiency in the restricted case too, whereas a portfolio efficient relative to a restricted portfolio possibilities set may very well be inefficient with respect to the same set with the short sales restriction relaxed. Therefore, the unrestricted case can be seen as a generalization of the restricted short sales and has a practical advantage of not having to specify the exact boundaries for short sales. Moreover, as we shall show further in this section, some algorithms proposed below have superior properties in terms of computational complexity relative to traditional methods in the case when no short sales are assumed. Finally, some of the efficiency tests are only applicable when short sales are restricted, for instance Post (2003) test assumes the portfolio possibilities set to be a polyhedron, so the formulation of the test explicitly includes the vertices of this set.

\subsection{SSD Efficiency}

Although many SSD efficiency tests have been proposed already (see Post (2003), Dentcheva and Ruszczynski (2003), Kuosmanen (2004), Post and Versijp (2007) among others) we shall focus on linear programming formulations only, since such methods have the lowest computational complexity, which is often a burden for real-life data sets, particularly when it comes to repeating the test many times for statistical inference and bootstrapping or high dimensionality of the data (see for instance Dentcheva and Ruszczynski, 2006). By far the least computationally demanding SSD efficiency test known is Post (2003). In this section we derive another LP test that is even more efficient than that of Post (2003) in the case when short sales are unrestricted. In the following section we derive a TSD efficiency test, also exploiting the special structure of portfolio possibilities sets and thereby improving its computational complexity.

Consider a given portfolio $x$. As the ordering of states of the world is not relevant, we may assume without loss of generality that $x$ is sorted in 
ascending order: $x_{1} \leq x_{2} \leq \cdots \leq x_{m}$. In order to determine if $x$ is SSD efficient, we need to find a supporting gradient vector $z \in \mathbb{R}^{m}$. First note, that condition (10a)

$$
(X \alpha)^{\top} z=C, \forall \alpha \in \Lambda
$$

is equivalent to $X^{\top} z=C e$. We are interested in the case when the market is incomplete and $m>n$. Without loss of generality we may assume that the first $n$ rows of $X$ are linearly independent. Partitioning $X$ into $X_{1}$ (first $n$ rows) and $X_{2}$ (the rest $(m-n)$ rows), we may write:

$$
X^{\top} z=\left[X_{1} X_{2}\right]^{\top} z=X_{1}^{\top} z_{1: n}+X_{2}^{\top} z_{n+1: m}=C e .
$$

Therefore, the general solution of (10a) can be expressed as

$$
z=\left[\begin{array}{c}
\left(X_{1}^{\top}\right)^{-1}\left(C e-X_{2}^{\top} \beta\right) \\
\beta
\end{array}\right]
$$

where $\beta$ is $(m-n)$-parameter vector. Since only the ordering of elements of $z$ matters, $z$ can be normalized, so that $C=1$.

Given the criteria above, the portfolio $x$ is efficient if and only if there exists a decreasing positive vector $z$ satisfying (11). If such $z$ exists, it is also a strictly interior point to the following set:

$$
\begin{gathered}
\left\{\beta \in \mathbb{R}^{m-n}: D\left[\begin{array}{c}
-\left(X_{1}^{\top}\right)^{-1} X_{2}^{\top} \\
I_{m-n}
\end{array}\right] \beta \leq-D\left[\begin{array}{c}
\left(X_{1}^{\top}\right)^{-1} e \\
0_{m-n}
\end{array}\right]\right\}, \\
\text { where } D=\left[\begin{array}{rrrrr}
-1 & 1 & 0 & 0 & 0 \\
0 & -1 & 1 & \ddots & 0 \\
0 & \ddots & \ddots & \ddots & 0 \\
0 & 0 & 0 & -1 & 1 \\
0 & 0 & 0 & 0 & -1
\end{array}\right] \cdot\left[\begin{array}{c}
\pi_{1}^{-1} \\
\pi_{2}^{-1} \\
\vdots \\
\pi_{m}^{-1}
\end{array}\right] .
\end{gathered}
$$

This test can be equivalently formulated as the following linear program:

$$
\max _{\beta \in \mathbb{R}^{m-n}, \theta \in \mathbb{R}}\left\{\theta:\left[\begin{array}{c}
-\left(X_{1}^{\top}\right)^{-1} X_{2}^{\top} \\
I_{m-n}
\end{array}\right] \beta+D\left[\begin{array}{c}
\left(X_{1}^{\top}\right)^{-1} e \\
0_{m-n}
\end{array}\right]+\theta \leq 0\right\} .
$$


Allocation $x$ is SSD efficient if and only if (13) is either unbounded or $\theta^{*}>0 .^{2}$

Efficiency test (13) is less computationally demanding than that of Post (2003), since (13) has $m-n+1$ variables and $m$ constraints, which is $n$ variables and $n^{2}$ non-zeros in the constraints matrix less than in Post (2003). By changing variables

$$
y_{i}=\pi_{n+i+1}^{-1} \beta_{i+1}-\pi_{n+i}^{-1} \beta_{i}, \quad i=1, \ldots, m-n-1, \text { and } y_{m-n}=-\pi_{m}^{-1} \beta_{m-n},
$$

one can transform (13) to the standard form

$$
\max \left\{c^{\top} y: A y \leq b, y \geq 0\right\}
$$

with an $n$-by- $(m-n)$ matrix of constraints. The number of non-zeros in this matrix is a good indicator of the computational complexity of a linear program (for instance, Performance World (2009) ranks linear programs based on this criterion). The test of Post (2003) in the same standard form will have an $n$-by- $m$ matrix of constraints, all the elements of which are generally non-zeros. The difference of $n^{2}$ non-zero elements confirms the computational advantage of (13) relative to Post (2003).

The computational advantage of (13) becomes particularly eminent when $n$ approaches $m$ and for instance in the case of bootstrapping, when the efficiency test has to be run many times on multiple data samples generated from the estimated joint distribution of asset returns. However, for large values of $n$ one needs to invert a larger $X_{1}$ prior to solving (13). Should $X$ happen to be particularly ill-conditioned, one may use the following equivalent test without decomposing $X$ :

$$
\max _{z \in \mathbb{R}^{m}, \theta \in \mathbb{R}}\left\{\theta: X^{\top} U d=e, d \geq 0\right\} .
$$

where $U$ is an upper triangular $m$-by- $m$ matrix adjusted by the probabilities of the states of nature, $d$ is an $m$-vector representing the probability-adjusted step differences of vector $z$, that is

$$
d_{j}=\pi_{j+1}^{-1} z_{j+1}-\pi_{j}^{-1} z_{j}, \quad j=1, \ldots, m-1, \text { and } d_{m}=\pi_{m}^{-1} z_{m} .
$$

\footnotetext{
${ }^{2}$ Equivalently, $x$ is SSD inefficient if $(13)$ is infeasible. The case $\theta^{*}=0$ also implies inefficiency (non-optimality) by our definition. Some authors consider portfolios corresponding to $\theta^{*}=0$ efficient as well. In this case the efficiency criterion can be easily adjusted without altering the computational complexity of (13).
} 
Program (14) has $m+1$ variables and $n$ equality constraints and is therefore similar to Post (2003) test in terms of computational complexity. However, the two tests are applicable in different circumstances: (13) and (14) apply when no short sales restrictions are postulated, whereas the test of Post (2003) requires the portfolio possibilities set to be bounded and to contain the subject portfolio $x$ in its interior.

\subsection{TSD Efficiency}

A portfolio $x^{0} \in M_{X}$ is Third Order SD (TSD) efficient if and only if there exists $u_{0} \in U_{3}$ such that $E u_{0}\left(x^{0}\right)=\sup _{x \in M_{X}} E u_{0}(x)$, where

$$
U_{3}=U_{2} \cap\left\{u: u^{\prime \prime \prime}(x)>0\right\} .
$$

Employing concavity of the first derivative of any function in $U_{3}$, it is straightforward to formulate TSD efficiency criteria: A portfolio $x^{0} \in M_{X}$ is efficient in $U_{3}$ if and only if there exists $z^{0} \in \mathbb{R}^{m}$ such that:

(i) $x^{\top} z^{0}$ is constant for all $x \in M_{X}$

(ii) $x_{i}^{0}<x_{j}^{0} \Rightarrow \frac{z_{i}^{0}}{\pi_{i}} \geq \frac{z_{j}^{0}}{\pi_{j}}, \forall i, j$

(iii) $z^{0}>0$

(iv) $x_{i}^{0}<x_{j}^{0}<x_{k}^{0} \Rightarrow \frac{z_{j}^{0}}{\pi_{j}} \leq \frac{z_{k}^{0}}{\pi_{k}}+\left(\frac{z_{i}^{0}}{\pi_{i}}-\frac{z_{k}^{0}}{\pi_{k}}\right) \frac{x_{k}^{0}-x_{j}^{0}}{x_{k}^{0}-x_{i}^{0}}, \quad \forall i, j, k$

The corresponding TSD efficiency test for a given portfolio also leads to a linear programming formulation. Indeed, the TSD criteria (15) is simply

$$
\begin{array}{r}
\bar{D}\left[\begin{array}{c}
-\left(X_{1}^{\top}\right)^{-1} X_{2}^{\top} \\
I_{m-n}
\end{array}\right] \beta \leq-\bar{D}\left[\begin{array}{c}
\left(X_{1}^{\top}\right)^{-1} e \\
0_{m-n}
\end{array}\right] \text {, where } \\
\bar{D}=\left[\begin{array}{cccccc}
a_{1} & b_{1} & c_{1} & 0 & 0 & 0 \\
0 & a_{2} & b_{2} & c_{2} & \ddots & 0 \\
0 & 0 & \ddots & \ddots & \ddots & 0 \\
0 & 0 & \ddots & a_{m-2} & b_{m-2} & c_{m-2} \\
0 & \ddots & 0 & 0 & a_{m-1} & b_{m-1} \\
0 & 0 & 0 & 0 & 0 & a_{m}
\end{array}\right], \text { with }\left\{\begin{array}{l}
a_{i[i=1 \ldots m-2]}=\frac{-1}{\pi_{i}\left(x_{i+2}-x_{i}\right)} \\
b_{i[i=1 \ldots m-2]}=\frac{1}{\pi_{i+1}\left(x_{i+2}-x_{i+1}\right)} \\
c_{i[i=1 \ldots m-2]}=\frac{1}{\pi_{i+2}}\left(\frac{1}{x_{i+2}-x_{i}}-\frac{1}{x_{i+2}-x_{i+1}}\right) \\
a_{m-1}=-\pi_{m-1}^{-1}, b_{m-1}=\pi_{m}^{-1} \\
a_{m}=-\pi_{m}^{-1}
\end{array}\right.
\end{array}
$$


System (16) can be solved for $\beta$ via the same program (13) that was applied to the SSD test, with redefined matrix $\bar{D}$. Therefore, the TSD efficiency test is a linear program with $m-n+1$ variables and $m$ constraints.

\subsection{Stochastic Dominance for Decreasing Absolute Risk Aversion (DSD)}

It is well accepted within expected utility framework that rational individuals possess decreasing absolute risk aversion (DARA). ${ }^{3}$ Let us examine Stochastic Dominance efficiency relative to this class of utility functions. Define

$$
U_{d}=U_{2} \cap\left\{\frac{d}{d x}\left(-\frac{u^{\prime \prime}(x)}{u^{\prime}(x)}\right)<0, \forall x\right\} .
$$

An allocation $x^{0} \in M_{X}$ is efficient in $U_{d}$ (DSD efficient) if and only if there exists $u_{0} \in U_{d}$ such that $E u_{0}\left(x^{0}\right)=\sup \left\{E u_{0}(x): x \in M_{X}\right\}$.

To express properties of the risk aversion in terms of supporting vectors, we have to adapt the efficiency criterion. Let $r(x)=-\frac{u^{\prime \prime}(x)}{u^{\prime}(x)}$ be the absolute risk aversion (ARA) of $u(x)$. We have: $u(x)=\exp \left(-\int r(x) d x+C\right)$, and if $x_{1}<x_{2}<\ldots<x_{n}$, then

$$
u^{\prime}\left(x_{i}\right)=u^{\prime}\left(x_{i-1}\right) \exp \left(-\int_{x_{i-1}}^{x_{i}} r(x) d x\right) .
$$

Therefore, it suffices to require that $r(x) \geq 0$ and $r^{\prime}(x)<0$ for all $x$ to ensure that $u \in U_{d}$. With such $r(x)$, the $\exp \left(-\int_{x_{i-1}}^{x_{i}} r(x) d x\right)$ is bounded by

$$
\exp \left(-r_{i-1}\left(x_{i}-x_{i-1}\right)\right) \leq \exp \left(-\int_{x_{i-1}}^{x_{i}} r(x) d x\right) \leq \exp \left(-r_{i}\left(x_{i}-x_{i-1}\right)\right) \text {. }
$$

Thus, there should hold:

$$
\exp \left(-r_{i-1}\left(x_{i}-x_{i-1}\right)\right) \leq \frac{u^{\prime}\left(x_{i}\right)}{u^{\prime}\left(x_{i-1}\right)} \leq \exp \left(-r_{i}\left(x_{i}-x_{i-1}\right)\right)
$$

\footnotetext{
${ }^{3}$ See e.g. Pratt (1964) for formal derivation and discussion.
} 
and therefore:

$$
r_{m} \leq \ldots \leq r_{i+1} \leq \frac{-\ln \left(\frac{u^{\prime}\left(x_{i+1}\right)}{u^{\prime}\left(x_{i}\right)}\right)}{x_{i+1}-x_{i}} \leq r_{i} \leq \frac{-\ln \left(\frac{u^{\prime}\left(x_{i}\right)}{u^{\prime}\left(x_{i-1}\right)}\right)}{x_{i}-x_{i-1}} \leq r_{i-1} \leq \ldots \leq r_{1} .
$$

Clearly, a decreasing sequence $\left\{r_{i}\right\}$ in (17) exists if and only if

$$
\frac{\ln \left(u^{\prime}\left(x_{i}\right)\right)-\ln \left(u^{\prime}\left(x_{i+1}\right)\right)}{x_{i+1}-x_{i}} \leq \frac{\ln \left(u^{\prime}\left(x_{i-1}\right)\right)-\ln \left(u^{\prime}\left(x_{i}\right)\right)}{x_{i}-x_{i-1}}, \text { for all } i=2, \ldots, m-1
$$

We are now ready to adapt the efficiency definition to the class of $U_{d}$. An allocation $x^{0} \in M_{X}$ is efficient in $U_{d}$ (DSD efficient) if and only if there exists $z^{0} \in \mathbb{R}^{m}$ such that:

(i) $x^{\top} z^{0}$ is constant for all $x \in M_{X}$

(ii) $x_{i}^{0}<x_{j}^{0} \Longrightarrow \frac{z_{i}^{0}}{\pi_{i}} \geq \frac{z_{j}^{0}}{\pi_{j}}, \forall i, j$

(iii) $z^{0}>0$

$$
\text { (iv) } x_{i}^{0}<x_{j}^{0}<x_{k}^{0} \Longrightarrow \frac{\ln \frac{z_{j}^{0}}{\pi_{j}}-\ln \frac{z_{k}^{0}}{\pi_{k}}}{x_{k}^{0}-x_{j}^{0}} \leq \frac{\ln \frac{z_{i}^{0}}{\pi_{i}}-\ln \frac{z_{j}^{0}}{\pi_{j}}}{x_{j}^{0}-x_{i}^{0}}, \quad \forall i, j, k
$$

Note that DSD efficiency implies TSD efficiency. This follows from the fact that $r^{\prime}(x)=u^{\prime}(x)^{-2}\left(u^{\prime \prime}(x)^{2}-u^{\prime}(x) u^{\prime \prime \prime}(x)\right)$. This is also consistent with DSD-TSD criteria (15) and (19): (19d) implies (15d), since the geometric average in $(19 \mathrm{~d})$ can not exceed the arithmetic average in (15d).

We are now ready to formulate a test for DSD efficiency of a given portfolio which will no longer be linear, but still a convex program. Indeed, any $z=\left[\begin{array}{c}-\left(X_{1}^{\top}\right)^{-1} X_{2}^{\top} \\ I_{m-n}\end{array}\right] \beta$ satisfying the DSD criteria (19) is also an interior point to the set $^{4}$

$$
\hat{D} \ln \left(\left[\begin{array}{c}
-\left(X_{1}^{\top}\right)^{-1}\left(e-X_{2}^{\top}\right) \\
I_{m-n}
\end{array}\right] \div \pi\right) \leq 0, \text { where }
$$

\footnotetext{
${ }^{4}$ By $y=\ln (x), x \in \mathbb{R}^{m}$, we mean the element-wise logarithm, that is, $y \in \mathbb{R}^{m}$ and $y_{i}=\ln \left(x_{i}\right)$. Similarly, $z=y \div x, x, y \in \mathbb{R}^{m}$, means $z \in \mathbb{R}^{m}$ s.t. $z_{i}=y_{i} / x_{i}$.
} 
$\hat{D}=\left[\begin{array}{cccccc}a_{1} & b_{1} & c_{1} & 0 & 0 & 0 \\ 0 & a_{2} & b_{2} & c_{2} & \ddots & 0 \\ 0 & \ddots & \ddots & \ddots & \ddots & 0 \\ 0 & \ddots & 0 & a_{m-2} & b_{m-2} & c_{m-2} \\ 0 & 0 & 0 & 0 & a_{m-1} & b_{m-1}\end{array}\right]$, with $\left\{\begin{array}{l}a_{i[i=1 \ldots m-2]}=\frac{-1}{x_{i+1}-x_{i}} \\ b_{i[i=1 \ldots m-2]}=\frac{1}{x_{i+2}-x_{i+1}}-\frac{1}{x_{i+1}-x_{i}} \\ c_{i[i=1 \ldots m-2]}=\frac{-1}{x_{i+2}-x_{i+1}} \\ a_{m-1}=-1, b_{m-1}=1\end{array}\right.$

Even though the constraints on $\beta$ are no longer linear, they are still convex, and therefore we can find strictly feasible points (or establish that they do not exist) efficiently.

\subsection{SD for Decreasing Absolute and Increasing Rela- tive Risk Aversion (DISD)}

In addition to DARA, relative risk aversion is often postulated to be increasing among rational individuals (see e.g. Pratt, 1964). In this section we examine optimality conditions in the utility class $U_{d i}$ combining the two risk aversion properties:

$$
U_{d i}=U_{d} \cap\left\{\frac{d}{d x}\left[-\frac{x u^{\prime \prime}(x)}{u^{\prime}(x)}\right] \geq 0, \forall x>0\right\}
$$

The utility functions under consideration are therefore those having decreasing absolute (DARA) and increasing relative risk aversion (IRRA). A portfolio $x^{0} \in M_{X}$ is said to be efficient in $U_{d i}$ (DISD efficient) if and only if there exists $u_{0} \in U_{d i}$ such that

$$
E u_{0}\left(x^{0}\right)=\sup \left\{E u\left(x^{0}\right): u \in U_{d i}\right\} .
$$

Given the ARA values $r_{i}$ and $r_{i+1}$ (such that $r_{i} \geq r_{i+1}$ ) at nodes $x_{i}$ and $x_{i+1}$, the IRRA requirement restricts $r(x)$ to lie above $^{5} f(x)=\frac{r_{i} x_{i}}{x}$, for $x_{i} \leq x \leq x_{i+1}$, imposing thereby an extra condition:

$$
r_{i} x_{i} \leq r_{i+1} x_{i+1}, \quad i=1, \ldots, m-1
$$

${ }^{5} f(x)$ is a limiting case of ARA in order for RRA to remain non-decreasing in the interval $\left[x_{i-1}, x_{i}\right]$. It is the solution of $\left\{(f(x) x)^{\prime}=0, f\left(x_{i}\right)=r_{i}\right\}$. 
Conversely, if (21) holds, we can always construct $f(x)=\frac{r_{i} x_{i}}{x}$, for $x_{i} \leq x \leq x_{i+1}$, such that $x r(x)$ will be non-decreasing, provided that

$$
\begin{aligned}
& r_{i+1} x_{i+1}+r_{i} x_{i}(\left.\ln \frac{r_{i}}{r_{i+1}}-1\right)=\int_{x_{i}}^{x_{i+1}} \max \left\{\frac{r_{i} x_{i}}{x}, r_{i+1}\right\} d x \leq \\
& \leq \int_{x_{i}}^{x_{i+1}} r(x) d x=\ln \frac{u^{\prime}\left(x_{i}\right)}{u^{\prime}\left(x_{i+1}\right)} \leq \int_{x_{i}}^{x_{i+1}} \min \left\{\frac{r_{i+1} x_{i+1}}{x}, r_{i}\right\} d x
\end{aligned}
$$

Therefore

$$
r_{i+1} x_{i+1}+r_{i} x_{i}\left(\ln \frac{r_{i}}{r_{i+1}}-1\right) \leq \ln \frac{u^{\prime}\left(x_{i}\right)}{u^{\prime}\left(x_{i+1}\right)} \leq r_{i+1} x_{i+1}\left(\ln \frac{r_{i}}{r_{i+1}}+1\right)-r_{i} x_{i} .
$$

This leads to the following DISD efficiency criterion: An allocation $x^{0} \in M_{X}$ is efficient in $U_{d i}$ (DISD efficient) if and only if there exist $z^{0}$ and $r \in \mathbb{R}^{m}$ such that:

(i) $x^{\top} z^{0}$ is constant for all $x \in M_{X}$

(ii) $x_{i}^{0}<x_{j}^{0} \Longrightarrow \frac{z_{i}^{0}}{\pi_{i}} \geq \frac{z_{j}^{0}}{\pi_{j}}, \forall i, j$

(iii) $z^{0}>0$

(iv) $x_{i}^{0}<x_{j}^{0}<x_{k}^{0} \Longrightarrow r_{k} \leq \frac{-\ln \frac{x_{k}^{0} \pi_{j}}{x_{j}^{0} \pi_{k}}}{x_{k}^{0}-x_{j}^{0}} \leq r_{j} \leq \frac{-\ln \frac{x_{j}^{0} \pi_{i}}{x_{i}^{0} \pi_{j}}}{x_{j}^{0}-x_{i}^{0}} \leq r_{i}$

(v) $x_{i}^{0}<x_{j}^{0} \Longrightarrow r_{j} x_{j}^{0}+r_{i} x_{i}^{0}\left(\ln \frac{r_{i}}{r_{j}}-1\right) \leq$

$$
\leq-\ln \frac{x_{j}^{0} \pi_{i}}{x_{i}^{0} \pi_{j}} \leq r_{j} x_{j}^{0}\left(\ln \frac{r_{i}}{r_{j}}+1\right)-r_{i} x_{i}^{0}
$$

The above condition is far less convenient than those for TSD or DSD, as both $r$ and $x$ are now entering (23d) and (23e) in both linear and logarithmic form. 


\section{Concluding remarks}

We have pointed out the importance of stochastic dominance efficient sets being convex and further summarized and extended conditions leading to convexity of efficient sets. This property has great importance both practically (passive vs. active investing strategies, efficiency of mutual funds) and theoretically (heterogeneous investors models and asset pricing) and can be analyzed from two different but interrelated approaches: the returns on underlying assets and the utilities of individual investors. Restricting distributions of returns typically leads to various factor models, where a complete class of non-satiable and risk-averse investors is assumed. Restricting the set of utilities can also affect efficient sets considerably, as can be seen, for example, in Rubinstein (1974). Unfortunately the extent to which restrictions on sets of utilities affect convexity has not been duly researched.

Based on the efficiency criteria (19), Dybving and Ross (1982) derive the following characterization of SSD efficient sets $E_{S S D}$ :

$$
E_{S S D}=\bigcup_{z \in Z} \bigcap_{(i, j): \frac{z_{i}}{\pi_{i}}<\frac{z_{j}}{\pi_{j}}}\left\{x \in M_{X}: x_{i}>x_{j}\right\},
$$

where the union is taken over all $z \in Z$ having different orderings $\left\{\frac{z_{j}}{\pi_{j}}\right\}$. Since the dimensionality of $z$ is $m$, the number of different orderings is at most $m$ !. Thus, $E_{S S D}$ is a union of a finite number of convex sets. By analogy, we can explicitly characterize TSD efficient sets:

$$
\begin{aligned}
E_{T S D}= & \bigcup_{z \in Z}\left(\bigcap_{(i, j): \frac{z_{i}}{\pi_{i}}<\frac{z_{j}}{\pi_{j}}}\left\{x \in M_{X}: x_{i}>x_{j}\right\}\right. \\
& \left.\bigcap_{(i, j, k): x_{i}<x_{j}<x_{k}}\left\{x \in M_{X}:\left(\frac{z_{j}}{\pi_{j}}-\frac{z_{k}}{\pi_{k}}\right)\left(x_{k}-x_{i}\right)<\left(\frac{z_{i}}{\pi_{i}}-\frac{z_{k}}{\pi_{k}}\right)\left(x_{k}-x_{j}\right)\right\}\right),
\end{aligned}
$$

Since all restrictions on $x$ are linear, $E_{T S D}$ is again a finite union of convex sets. The same applies for DSD: 


$$
\begin{aligned}
E_{D S D}= & \bigcup_{z \in Z}\left(\bigcap_{(i, j): \frac{z_{i}}{\pi_{i}}<\frac{z_{j}}{\pi_{j}}}\left\{x \in M_{X}: x_{i}>x_{j}\right\}\right. \\
& \left.\bigcap_{(i, j, k): x_{i}<x_{j}<x_{k}}\left\{x \in M_{X}: \ln \left(\frac{z_{j} \pi_{k}}{z_{k} \pi_{j}}\right)\left(x_{j}-x_{i}\right)<\ln \left(\frac{z_{i} \pi_{j}}{z_{j} \pi_{i}}\right)\left(x_{k}-x_{j}\right)\right\}\right),
\end{aligned}
$$

The DISD efficiency characterization is slightly more complex, as $r$ appears along with $z$ :

$$
\begin{aligned}
E_{D I S D}= & \bigcup_{\substack{z \in Z, Z \\
r \in \mathbb{R}_{+}^{m}}}\left(\bigcap_{\substack{(i, j): \frac{z_{i}}{\pi_{i}}<\frac{z_{j}}{\pi_{j}}\\
}}\left\{x \in M_{X}: x_{i}>x_{j}\right\}\right. \\
& \bigcap_{\substack{(i, j, k): x_{i}<x_{j}<x_{k}, x \in M_{X}}}\left\{r_{k} \leq \frac{-\ln \frac{x_{k} \pi_{j}}{x_{j} \pi_{k}}}{x_{k}-x_{j}} \leq r_{j} \leq \frac{-\ln \frac{x_{j} \pi_{i}}{x_{i} \pi_{j}}}{x_{j}-x_{i}} \leq r_{i}\right\} \\
& \left.\bigcap_{\substack{(i, j): x_{i}<x_{j}, x \in M_{X}}}\left\{r_{j} x_{j}+r_{i} x_{i}\left(\ln \frac{r_{i}}{r_{j}}-1\right) \leq-\ln \frac{x_{j} \pi_{i}}{x_{i} \pi_{j}} \leq r_{j} x_{j}\left(\ln \frac{r_{i}}{r_{j}}+1\right)-r_{i} x_{i}\right\}\right) .
\end{aligned}
$$

It is not clear whether $E_{D I S D}$ is necessarily non-convex. On the other hand, it is difficult to find a general set of assumptions that would guarantee convexity of a union of convex sets, in contrast to the intersection of convex sets which is automatically convex.

With regard to the link between utility functions and convexity of efficient sets, there are only extreme cases known so far: when investors are nearly homogeneous (as in Rubinstein (1974)), in which case efficient sets are normally rays or lines, and when investors' preferences are spanned unrealistically broadly, such as the whole $U_{2}$, where efficient sets are too large (even without portfolio restrictions on short sales) and non-convex.

A possible extension of the current research could lie in searching for a reasonable set of well-behaved utility functions for which the efficient sets 
would be large enough and convex. In analogy with arbitrage pricing theories and factor models for returns, one could try to parameterize investors' preferences. The expo-power utility function of the form

$$
u(x)=\theta-\exp \left(-\beta x^{\alpha}\right),
$$

where $\theta>1, \alpha \neq 0, \beta \neq 0$, and $\alpha \beta>0$, seems to be suitable for that, as it allows all possible combinations of absolute (increasing, decreasing or constant) and relative (increasing or decreasing) risk aversion with only two key parameters.

In addition to the convexity analysis, we have also derived some higher order stochastic dominance efficiency tests in which we incorporate some meaningful restrictions on the set of utilities well recognized in the expected utility framework, such as decreasing absolute and increasing relative risk aversion.

\section{References}

[1] BAWA V. S., Stochastic Dominance: A Research Bibliography, Management Science, 28, 1982, pp. 698-712.

[2] BIXBY R. E., Solving real-world linear programs: A decade and more of progress. Oper. Res. 2002, 50(1) 3-15.

[3] COCHRAne J. H., Asset Pricing, 2nd Ed, Princeton University Press, 2005.

[4] DENTCHEVA D., RUSZCZYŃSKI A., Optimization with Stochastic Dominance Constraints, SIAM Journal on Optimization, 14 (2003) pp. 548-566.

[5] DENTCHeVA D., RUSZCZYŃSKI A., Portfolio Optimization with Stochastic Dominance Constraints, Journal of Banking and Finance, 30/2 (2006), pp. 433-451.

[6] DYBVIG P. H., ROSS S. A., Portfolio Efficient Sets. Econometrica, Vol. 50, No. 6. (Nov., 1982), pp. 1525-1546.

[7] EECKHOUDT L., SCHLESINGER H., TSETLIN I., Approximating of Risks via Stochastic Dominance, Journal of Economic Theory, 144-3, May 2009, pp. 994-1003. 
[8] KUOSMAnEN T., Efficient Diversification According to Stochastic Dominance Criteria, Management Science, Vol. 50, No. 10, 2004, pp. 1390-1406.

[9] LEVY H., Stochastic Dominance And Expected Utility: Survey And Analysis, Management Science, Vol. 38, No. 4 (1992).

[10] LEVY H., Stochastic Dominance: Investment Decision Making under Uncertainty, Second Edition, Springer Science + Business Media, inc., 2006.

[11] LINTER J., The aggregation of Investor's Diverse Judgments and Preferences in Purely Competitive Security Markets, The Journal of Financial and Quantitative Analysis, Vol. 4, No. 4. (Dec. 1969), pp. 347-400.

[12] MARKOWITZ H. M., Portfolio Selection, The Journal of Finance, 7 (1952), pp. 77-91

[13] MARKOWITZ H. M., Mean Variance Analysis In Portfolio Choice And Capital Markets, Basil Blackwell, New York, 1978.

[14] PERFORMANCE WORLD (viewed October 2009). GAMS Development Corp. http://www.gamsworld.org/performance/plib/size.htm.

[15] POST T., Empirical Tests for Stochastic Dominance Efficiency, The Journal of Finance, Vol. 58, No. 5. (Oct., 2003), pp. 1905-1931.

[16] POST T., VERSIJP P., Multivariate Tests for Stochastic Dominance Efficiency of a Given Portfolio, JFQA 42-2 (June 2007), pp. 489-516.

[17] PRATT J. W., Risk Aversion in the Small and in the Large. Econometrica, Vol. 32, No. 1/2 (Jan. - Apr. 1964), pp. 122-136.

[18] ROSS S. A., Mutual Fund Separation in Financial Theory - The Separating Distributions, Journal of Economic Theory 17, pp. 254-286 (1978).

[19] RUBINSTEIN M., An Aggregation Theorem For Securities Markets. Journal of Financial Economics I (1974) pp. 225-244.

[20] VERSIJP P., Advances in the use of stochastic dominance in asset pricing, Tinbergen Institute PhD Thesis \# 407, Thela Thesis, 2007. 